\title{
Broad Ligament Pregnancy: Lessons Learnt
}

\author{
${ }^{1}$ Preet Agarwal, ${ }^{2}$ Usha Viswanath, ${ }^{3}$ Rukshana, ${ }^{4}$ MS Natarajan
}

\begin{abstract}
Abdominal pregnancy is a rare obstetric condition seen by the obstetricians. Its diagnosis is usually difficult and management is a matter of debate. A 39-year-old multigravida with 20 weeks gestation complicated by pregnancy-induced hypertension, diabetes mellitus and anemia with previous cesarean section was admitted. She was not aware of her pregnancy earlier and she desired termination of pregnancy. But, termination of pregnancy failed inspite of using all possible methods of inducing labor. Diagnosis of abdominal pregnancy was made on by transvaginal ultrasound and proceeded with laparotomy. Left broad ligament pregnancy was found and the sac with fetus extracted. However, due to uncontrollable bleeding from broad ligament during placenta separation and hysterectomy was proceeded in this lady. We present our experience with the diagnosis and management of this rare case.
\end{abstract}

Keywords: Broad ligament pregnancy, Hemoperitoneum, Laparotomy.

How to cite this article: Agarwal P, Viswanath $U$, Rukshana, Natarajan MS. Broad Ligament Pregnancy: Lessons Learnt. J South Asian Feder Obst Gynae 2014;6(3):178-179.

\section{Source of support: Nil}

Conflict of interest: None declared

\section{INTRODUCTION}

Abdominal pregnancy is usually never seen by obstetricians during their career. It is a rare form of ectopic pregnancy and a life-threatening condition. Incidence of abdominal pregnancy is 10 in 1,00,000 live births. ${ }^{1}$ It is always diagnosed at an advanced stage or goes undetected till term. A pregnancy in the liver, sigmoid colon, spleen, diaphragm, pancreas, obturator foramen, peritoneum of pelvis have also been reported. ${ }^{3,4}$ Laparotomy for abdominal pregnancy is among the most difficult and dangerous operations as severe uncontrollable hemorrhage occurs during the separation of placenta. ${ }^{5}$ This is

\footnotetext{
${ }^{1}$ Associate Professor, ${ }^{2,4}$ Professor, ${ }^{3}$ Assistant Professor

${ }^{1-3}$ Department of Obstetrics and Gynecology, Sri Ramachandra University, Chennai, Tamil Nadu, India

${ }^{4}$ Department of Urology, Sri Ramachandra University, Chennai Tamil Nadu, India
}

Corresponding Author: Preet Agarwal, F-31, H Block, Jains Avantika Apartments, Manapakkam, Chennai-600125, Tamil Nadu, India, Phone: 9840447495, e-mail: preetagarwal3@ gmail.com a case report of a broad ligament pregnancy diagnosed during laparotomy following a failed medical termination of pregnancy. This case is reported for its rarity.

\section{CASE REPORT}

A 39-year-old multigravida with a previous live issue from a cesarean section (primigravida breech with pregnancy-induced hypertension (PIH) was referred from a private hospital as a case of 20 weeks $R$ h negative pregnancy with PIH with ultrasound showing retroplacental clots. Patient was not aware of her pregnancy as her cycles were irregular. She had giddiness on and off for 2 days prior to admission. She had a miscarriage 2 years earlier and she was also not sure of administration of anti-D after dilatation and curettage (D\&C).

Her uterus was 20 weeks, relaxed with a pulse rate of 128 beats per minute and blood pressure of 150/100 $\mathrm{mm} \mathrm{Hg}$ and with pedal edema. Urine albumin was negative. Her hemoglobin was $6.6 \mathrm{gm} / \mathrm{dl}$ and her indirect coombs test was positive (1:16 dilution).

Ultrasound was done which showed a live intrauterine pregnancy corresponding to 20 weeks gestation with moderate maternal ascites and umbilical artery Doppler showing high resistance flow. She was transfused with 3 units of packed cells. Termination of pregnancy was planned for social reasons.

She was administered tab. mifepristone $600 \mathrm{mg}$ orally and induced with four doses of prostaglandin (PGE2) gel and with seven doses of vaginal misoprostol 200 to 400 microgram $(\mathrm{mcg})$ every 4 hours. Cervix remained unfavorable even after mechanical dilation of cervix with intrauterine Foley's catheter for 24 hours. Hysterotomy was planned the next day.

Having the suspicion of abdominal pregnancy, a transvaginal ultrasound and saline sonography was done which revealed an empty uterine cavity and an amniotic sac with a live fetus posterior to the uterus with hemoperitoneum.

Emergency laparotomy was proceeded under general anesthesia with a vertical incision. Hemoperitoneum and huge clots adherent to omentum were removed. Right fallopian tube and ovary were normal. Gestational sac with fetus was found lying in pouch of Douglas (Fig. 1). Gestational sac was punctured during examination of posterior side of uterus and fetus was removed. Placenta was delivered as piece meal. Profuse bleeding was noted 


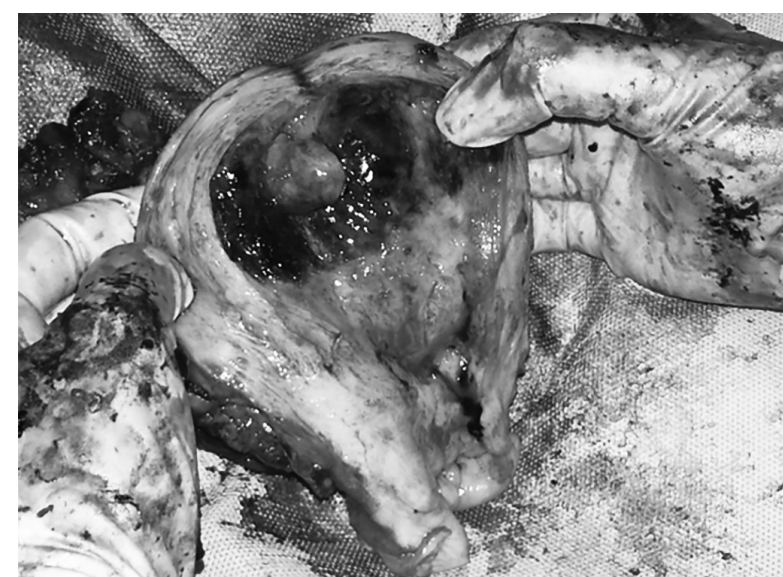

Fig. 1: Empty uterine cavity

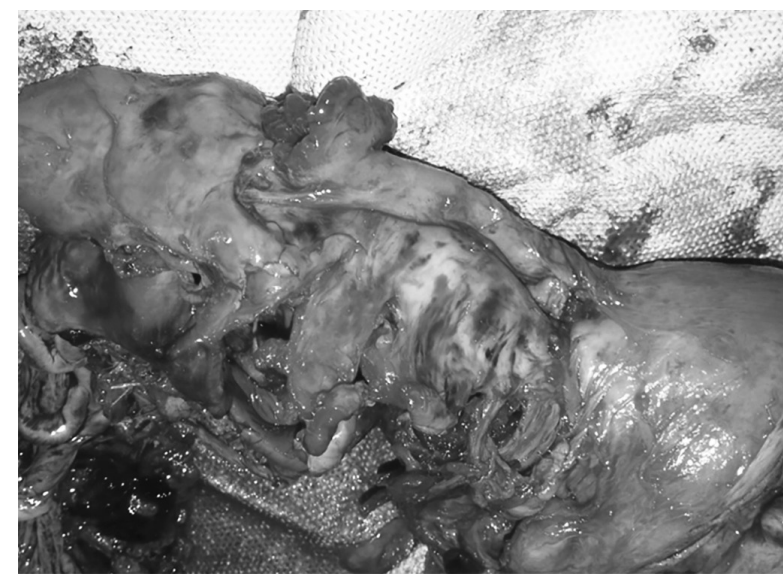

Fig. 3: Uterus with broad ligament

from the broad ligament and hence proceeded with hysterectomy. Intraoperatively, she had 2.2 liters of blood loss and was transfused with 4 units of packed cells and 4 units of fresh frozen plasma. Potassium was corrected intraoperatively and two abdominal drains (cul de sac and paracolic gutter) were placed before closure.

Cut section of uterus showed an empty cavity with broad ligament tear and intact right ovarian tissue (Fig. 2). Histopathology report revealed a damaged left fallopian tube and with intact ovary (Fig. 3). This confirmed the diagnosis of secondary abdominal pregnancy.

During postoperative period, patient had dribbling of urine from vagina. Computed tomography (CT) program showed transection of left ureter at the level of S2. Percutaneous nephrolithotomy was done in left side and prophylactic ureteric stenting in the right side. Later after 6 weeks stent was removed and she recovered.

\section{DISCUSSION}

Abdominal pregnancy accounts for $1 \%$ of ectopic pregnancies. Broad ligament pregnancy is either due to

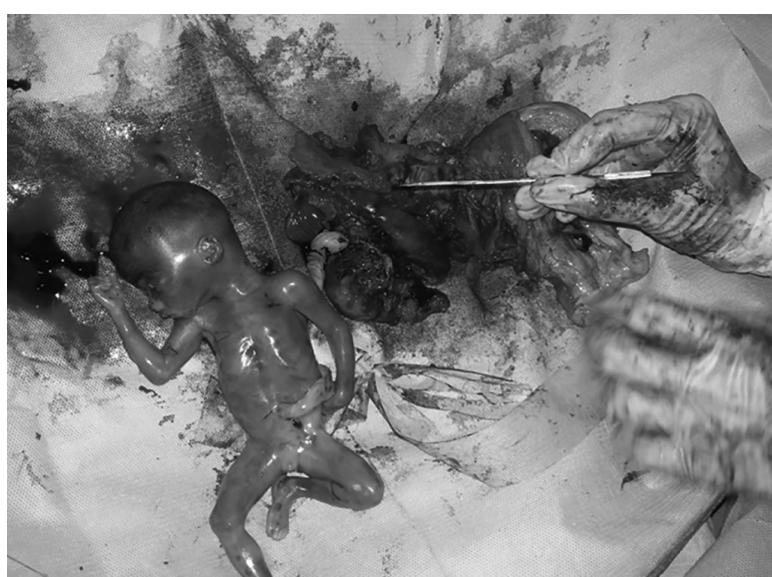

Fig. 2: Fetus with placenta and empty uterine cavity

primary implantation or secondary implantation after ruptured tubal or ovarian pregnancy.

This could be a ruptured tubal pregnancy followed by implantation in the broad ligament as confirmed by the fact that the bowels were intact without any placental adhesions and the histopathology report which revealed a damaged left tube and intact ovary.

The operation has to be quick and safe as possible. ${ }^{5}$ Management of placenta is usually a matter of debate in abdominal pregnancy ${ }^{2}$ and bleeding from placental site is always unpredictable.

Hysterectomy was unavoidable in this case as extraction of the gestational sac resulted in uncontrollable bleeding from broad ligament.

\section{CONCLUSION}

Abdominal pregnancy should be ruled out in any unexplained anemia or hemoperitoneum or failed medical termination of pregnancy. Such cases should be managed only in a tertiary care setting with adequate blood products at hand.

We should always have a high degree of suspicion, especially in our case where there was no response to medical management.

\section{REFERENCES}

1. Sheikh, et al. Abdominal pregnancy as a cause of hemoperitoneum. J Emerg Trauma Shock 2009 Sep-Dec;2(3):196-198.

2. Kun, et al. Abdominal pregnancy presenting as a missed abortion. HKMJ 2000;6(4):425.

3. Ganeshselvi, et al. Primary abdominal pregnancy implanted on the sigmoid colon. J Obstet Gynaecol 2003;23(6):667.

4. Chui AK, et al. Primary hepatic pregnancy. ANZ J Surg 2001; 71(4):260-261.

5. Marriott C. Specimen from case of extra-uterine pregnancy. Proceedings of the Royal Society of Medicine; 1919;12. 